\title{
MicroRNA Sequencing
}

National Cancer Institute

\section{Source}

National Cancer Institute. MicroRNA Sequencing. NCI Thesaurus. Code C156057.

A next-generation or massively parallel high-throughput DNA sequencing-based

procedure that can identify and quantify the microRNA sequences present in a biological sample. 